\title{
RELEVANCIA DEL ANÁLISIS DEL CONTEXTO COMPETITIVO EN LOS APORTES SOCIALES DE LAS EMPRESAS
}

\author{
Magdalena Aninat \\ Universidad Adolfo Ibáñez \\ Isidora Fuenzalida \\ Consultora independiente
}

\begin{abstract}
Resumen: Considerando que hoy las empresas aportan el 84 por ciento de las donaciones registradas en Chile, es relevante que las contribuciones sociales corporativas generen valor para la sociedad. La literatura destaca que la adopción de un enfoque estratégico en los aportes sociales corporativos permite alinear la competitividad de las empresas con la generación de beneficios sociales de mayor valor, al comprometer al donante en una mirada de largo plazo. Este enfoque permite, también, concebir el ámbito de la acción filantrópica como parte de las dimensiones sociales del contexto en que participa. Mediante el uso de una encuesta, este artículo comprueba que sí existe una correlación entre el análisis del contexto competitivo
\end{abstract}

Magdalena Aninat. Periodista de la Universidad Católica. Directora del Centro de Filantropía e Inversiones Sociales (Cefis) de la Escuela de Gobierno de la Universidad Adolfo Ibáñez (UAI). Email: magdalena.aninat@uai.cl.

Isidora Fuenzalida. Socióloga de la Universidad Católica. Coordinadora de investigación del Cefis UAI hasta junio de 2018, rol con el que fue coautora de este artículo. Email: isidorafuenzalida@gmail.com.

Las autoras agradecen los comentarios y sugerencias de José Luis Opazo, Verónica Devenin y de los dos árbitros anónimos de Estudios Públicos. Las autoras señalan que son las únicas responsables de las omisiones y errores del artículo. 
para la definición de los aportes sociales corporativos y la adopción de un enfoque estratégico en la filantropía corporativa. Asimismo, permite identificar que existe una tendencia positiva entre empresas que realizan análisis de contexto competitivo para definir sus aportes sociales e indicadores de valorización de mercado.

PALABRAs Clave: filantropía, responsabilidad social de la empresa, filantropía estratégica, análisis del contexto, beneficio social.

RECIBIDO: abril 2018; ACEPTADO: junio 2018.

Clasificación JEL: D64; L19; M14.

\title{
THE IMPORTANCE OF COMPETITIVE ENVIRONMENT ANALYSIS FOR FIRMS' SOCIAL CONTRIBUTIONS
}

\begin{abstract}
Considering that businesses currently account for 84 per cent of recorded donations in Chile, it is important that corporate social contributions should generate value for society. The literature points out that adopting a strategic approach to corporate social contributions makes it possible to align firms' competitiveness with the generation of higher-value social benefits by engaging the donor on a long-term view. This approach also means that the sphere of philanthropic action can be conceived as one of the social dimensions of the environment in which the firm operates. Using survey information, this article shows that there is a correlation between competitive environment analysis for the purposes of decision-making about corporate social contributions and adoption of a strategic approach to corporate philanthropy. It also reveals that there is a positive correlation between firms' carrying out competitive environment analyses to determine their social contributions and market valuation indicators.
\end{abstract}

KEYwords: philanthropy, corporate social responsibility, strategic philanthropy, context analysis, social benefit.

RECEIVED: April 2018; ACCEPTED: June 2018.

JEL CLASSIFICATION: D64; L19; M14.

\section{INTRODUCCIÓN}

n las últimas décadas, las empresas han destinado crecientes recursos a mejorar las relaciones con sus distintos grupos de interés, desarrollando inversiones sociales y reportes sobre sus aportes al bienestar social (Wang et al. 2008, 143). En este sentido se ha visto ampliado el rol empresarial de la mera provisión de bienes y servicios, o de fuente de empleo. El sector empresarial ha ido respondiendo a 
una expectativa pública que, desde los años sesenta, ha aumentado la exigencia a asumir una mayor responsabilidad en el bienestar de sus trabajadores, comunidades y otros stakeholders, incluso si ello significa sacrificar parte de las utilidades obtenidas (Carroll y Shabana 2010, 85). En este escenario surgió la responsabilidad social empresarial, que en su concepto amplio entiende que la empresa, como parte integral de la sociedad, requiere de consentimiento público para operar $\mathrm{y}$, por ende, tiene la responsabilidad de servir constructivamente a las necesidades de la sociedad (Van Marrewijk 2003, 97). Posteriormente, el término dio pie a una serie de conceptos relacionados, como el de sostenibilidad corporativa, que emerge hacia el año 2000, y otros como ciudadanía corporativa, ética empresarial, o triple bottom line. Estas expresiones, con sus diferencias específicas, se sobreponen o enmarcan en el de responsabilidad social empresarial, ya que se refieren, en general, a actividades voluntarias que demuestran la inclusión de aspectos sociales y medioambientales en la operación del negocio y en la interacción con sus grupos de interés, a la vez que integran temas de fondo, como valor, balance y rendición de cuentas (Van Marrewijk y Werre 2003, 107; Schwartz y Carroll 2008, 172).

Sin embargo, la discusión respecto de la pertinencia de este tipo de práctica en las empresas distingue dos tipos de posturas. Por una parte, en 1970 Milton Friedman señaló que "la única responsabilidad de las empresas es generar utilidades para sus accionistas cumpliendo las normas éticas y legales para ello" (Friedman 1970). Destinar parte de los recursos de la empresa a ámbitos del desarrollo social, señaló Friedman, atentaba contra la libertad de los accionistas de definir el destino de ellos. En esa misma línea, otros autores han señalado que adoptar políticas sociales y medioambientales puede destruir valor para los accionistas, debido a un costo de agencia, donde los ejecutivos reciben beneficios privados por adoptar estas políticas (prestigio personal, redes) pese a los costos que implica para la organización (Eccles et al. 2011, 2835 y 2853).

Otra línea argumental ha sostenido que es posible desarrollar modelos que permitan cumplir la máxima do well by doing good - tener buenos resultados haciendo el bien- (Godfrey 2005, 777; Porter y Kramer 2002, 3), identificando diversos efectos positivos que justificarían la adopción de políticas de sostenibilidad y el desarrollo de contribuciones sociales por parte de las empresas. Se ha señalado que 
estas políticas tienen un efecto en lograr una mayor atracción de talento y mayor productividad de sus colaboradores, mayor atracción de capital de inversión y de clientes y, en general, una percepción positiva de sus stakeholders, creando un capital moral para la empresa, que asegura ventaja competitiva y le permite mitigar la disposición a sancionarla ante eventos negativos (Smith 2003, 61-63; Godfrey 2005, 789).

Esta discusión sobre la pertinencia o no de la empresa de realizar acciones de responsabilidad social empresarial ha observado con especial énfasis el tema de la filantropía corporativa (por utilizar el término más extendido en la literatura). Carroll propone un modelo piramidal para definir el concepto de responsabilidad social corporativa: las responsabilidades filantrópicas ("contribuir con recursos a la comunidad y mejorar su calidad de vida") están situadas en los estamentos más altos de los que componen la pirámide de la responsabilidad social corporativa, seguidas por las responsabilidades éticas, legales y económicas situadas en orden descendente como base de la pirámide (Carroll 1991, 42). De este modo, el autor plantea que las responsabilidades filantrópicas corresponden al componente más discrecional y voluntario de la responsabilidad social empresarial.

Pese a esta discusión, en el mundo académico y empresarial - tanto a nivel internacional como en Chile - ha crecido la adhesión a reconocer la responsabilidad social empresarial como factor relevante para asegurar la sostenibilidad y también la rentabilidad empresarial, articulando el business case bajo cuatro líneas de argumentos: reducir costos y riesgos; fortalecer la legitimidad y reputación; construir ventajas competitivas, $\mathrm{y}$, por último, crear espacios de ventaja mutua —win-win- a través de una creación sinérgica de valor (Kurucz et al. 2008, 85-92). Sin embargo, diversos autores han hecho notar que, dados los costos que genera la responsabilidad social empresarial o la adopción de la visión de sostenibilidad, incluyendo su elemento discrecional (filantropía corporativa), el impacto sobre el negocio no siempre es positivo y sólo generará valor a la empresa en el tiempo en la medida en que se realice en forma estratégica y en su nivel óptimo (Wang et al. 2008, 144; Eccles et al. 2011, 2854). Ello requiere invertir los recursos - donaciones, conocimiento, voluntariado, redes - en aquellas acciones que demuestran una convergencia entre los objetivos económicos de la firma y los objetivos 
sociales de sus stakeholders (Kurucz et al. 2008, 91; Thorne y Ferrell 2002, 690; Carroll y Shabana 2010, 100).

Definir una estrategia de aportes sociales, que genere beneficio significativo para la sociedad y al mismo tiempo valor para el negocio, ocurre en la medida en que se logra definir el dónde y el cómo correctos, creando un círculo virtuoso entre las actividades filantrópicas y la ventaja competitiva (Porter y Kramer 2002, 66). La literatura señala que esta visión implica seleccionar como ámbito de acción filantrópica aquel o aquellos que sean parte de las dimensiones sociales de su contexto competitivo; es decir, enfocarse en factores sociales relevantes de su entorno, que afecten significativamente elementos subyacentes de la competitividad del negocio en las áreas en que la compañía opera (Porter y Kramer 2006, 6). Por lo mismo, cada estrategia será única para cada empresa, según su contexto competitivo (Smith 2003, 67). En esta línea, identificar los puntos de intersección bajo una mirada de mutua dependencia entre empresa y sociedad requiere, en primer lugar, realizar un análisis interno-externo (revisión del impacto social de la cadena de valor $)^{1}$ y un segundo nivel de análisis externo-interno respecto de las dimensiones sociales que afectan el contexto competitivo de la empresa (Porter y Kramer 2006, 5-9).

En la última década, diversos estudios han mostrado, a nivel internacional, una creciente adopción de la filantropía corporativa a nivel estratégico (Thorne y Ferrell 2002, 692; Carroll y Shabana 2010, 102), proceso en el cual las firmas deben adaptar aspectos organizacionales internos para su implementación (Brammer et al. 2006, 236). ${ }^{2}$ Esta adopción del enfoque estratégico favorece el posicionamiento de la empresa a ojos de sus stakeholders (File y Prince 1998, 1.529; Saiia et al. 2003, 186) y tiene efectos también en su desempeño financiero. Eccles et al.

${ }^{1}$ En esta misma línea, Porter y Kramer acuñaron posteriormente el concepto de "valor compartido", en el cual plantean que el propósito de las empresas debe ser redefinido y enfocarse en las conexiones entre el progreso económico y el de la sociedad, encontrando oportunidades de crecimiento para las empresas y también mayores beneficios para la sociedad, al reconcebir productos y mercados, redefinir la productividad en la cadena de valor y el desarrollo de un cluster local (Porter y Kramer 2011, 4-5).

${ }^{2}$ El estudio de Brammer et al. (2006) analiza elementos de implementación que determinan la filantropía estratégica en base a un análisis en empresas abiertas a la Bolsa de Londres. 
(2011) han explorado en Estados Unidos los positivos efectos financieros en las empresas con altos niveles de sostenibilidad, mientras Wang et al. han dado cuenta de los efectos positivos para la rentabilidad empresarial en empresas con un nivel óptimo de filantropía corporativa (Wang et al. 2008). Asimismo, otros autores han identificado los efectos positivos en la productividad y en la percepción de los stakeholders (Smith 2003, 61-63; Godfrey 2005, 789).

En Chile es posible identificar una creciente adhesión al concepto de responsabilidad social y de sostenibilidad empresarial. ${ }^{3}$ Junto con ello, los aportes sociales corporativos son especialmente relevantes para el ecosistema local: en las donaciones registradas ante el Servicio de Impuestos Internos, las empresas - principalmente las grandes - aparecen como las responsables del 84 por ciento de los recursos (Irarrázaval et al. 2017, 32-35). Sin embargo, un estudio reciente del Cefis UAI muestra que, entre las empresas que registran aportes sociales, sólo el 17 por ciento lo hace con un enfoque estratégico; esto es, seleccionan las áreas de destino de su donación porque están relacionadas a elementos del contexto competitivo o cadena de valor de la empresa (Aninat y Fuenzalida 2017, 46).

Pese a su relevancia, el desarrollo de la investigación académica sobre la adopción de enfoque estratégico es aún reducido, como también lo es la identificación específica de factores que facilitan su implementación para lograr la confluencia entre ventaja competitiva y valor social.

A partir de un análisis de la práctica empresarial, este artículo contribuye a la investigación existente, en la medida en que explora la rela-

${ }^{3}$ En Chile también ha existido una instalación del concepto de sostenibilidad en las últimas décadas, reemplazando el concepto de responsabilidad social empresarial por una visión más integral hacia las variables ambientales, sociales y de gobernanza (llamadas aspectos ASG). De hecho, se ha desarrollado una serie de acciones en este sentido: la creación del Dow Jones Sustainability Chile Index (DJSI Chile) en la Bolsa de Comercio de Santiago, que en su segunda versión, de 2016, incluyó a 21 compañías; la instalación en Chile de la iniciativa del Pacto Global de las Naciones Unidas; la constitución del Consejo de Responsabilidad Social para el Desarrollo Sostenible en el Ministerio de Economía, y la Norma de Carácter General n. 385 para Sociedades Anónimas Abiertas del año 2015, que obliga a publicar las prácticas de gobierno corporativo, responsabilidad social y desarrollo sostenible, de forma que los inversionistas tengan suficiente información para tomar decisiones y remitirlas a la SVS (Aninat y Fuenzalida 2017, 13). 
ción entre el análisis del contexto competitivo en la toma de decisiones de los aportes sociales y la implementación de un enfoque de filantropía estratégica, reafirmando una relación positiva y entregando con ello luces para implementar un enfoque que genere un mayor valor social, alineado con el desarrollo empresarial. Junto con ello, el artículo indaga en la relación entre análisis del contexto competitivo para los aportes sociales y la valorización de mercado de las empresas, aportando nuevos elementos para desarrollar una relación integrada entre empresa y sociedad.

En primer lugar, se establece el marco conceptual. En éste se examinan los enfoques en la filantropía corporativa y los conceptos de la literatura que permiten identificar la relevancia del análisis del contexto competitivo para el desarrollo de un enfoque estratégico en los aportes sociales de las empresas. Luego, se plantean dos hipótesis, las que son testeadas sobre los resultados de una encuesta aplicada a la unidad de análisis (empresas que transan en la Bolsa de Santiago y que registran aportes sociales entre los años 2013 y 2015), utilizando, para ello, un estudio factorial exploratorio, validado mediante otro de fiabilidad. Finalmente, los resultados presentados son discutidos y se identifican temas relevantes para futuras investigaciones.

\section{MARCO CONCEPTUAL}

La organización empresarial debe respetar la responsabilidad fiduciaria de sus inversionistas, pero también, como institución social legítima, tiene un rol crítico que jugar en la mantención de la infraestructura social en la que se desarrolla. En palabras del empresario colombiano Manuel Carvajal Sinisterra: "No puede haber una empresa sana en un medio social enfermo, porque tarde o temprano los males del medio repercuten en su desempeño. Por eso, el empresario responsable debe necesariamente comprometerse en la solución de los problemas sociales" (1960). ${ }^{4}$

Se ha señalado que la filantropía estratégica — término acuñado por Post y Waddock en 1995- es un oxímoron: ¿cómo una empresa puede impulsar sus intereses estratégicos, de creación de riqueza, al tiempo que dona recursos que aparentemente no le entregan nada a

\footnotetext{
${ }^{4}$ Véase http://www.fundacioncarvajal.org.co/lo-que-nos-inspira/.
} 
cambio? (Godfrey 2005, 779). La filantropía estratégica se define como la donación de recursos empresariales para abordar asuntos de la comunidad que no son propios del negocio, pero que también benefician la posición estratégica de la empresa y, en último término, su rentabilidad (Saiia et al. 2003, 170). Autores que adhieren a este concepto destacan que, pese a que la empresa no recibe a cambio un valor tangible o cuantificable en el corto plazo, las actividades filantrópicas y de responsabilidad social empresarial generan activos intangibles estratégicos que permiten favorecer el ambiente institucional para el desarrollo empresarial (Porter y Kramer 2002, 2).

De este modo, la filantropía estratégica emerge como el camino para aquella empresa que busca lograr un resultado sinérgico, al enfocar recursos corporativos a problemas sociales o temáticas que resuenan con los valores claves y la misión de la empresa. No sólo pone el acento en el beneficio para el negocio, sino que además tiene la fortaleza de maximizar el aporte que entrega a la sociedad, al incorporar una mirada de largo plazo y al involucrar integralmente al negocio en todos sus recursos (en el entendido de que una compañía dispone de distintos recursos: financieros, pero también tiempo, redes y expertise o conocimiento). En este enfoque, el acento está en el impacto social logrado en el tiempo, antes que en la comunicación inmediata de sus resultados. "La prueba de fuego de la buena filantropía corporativa - señalan Porter y Kramer $(2002,12)$ - es que el deseo de cambio social es tan beneficioso para la compañía que la empresa persistirá en lograr ese cambio, incluso si nadie sabe de ello".

Se opone entonces al enfoque altruista, entendido como donar sin esperar recompensa (Burlingame y Frishkoff, 1996, en Saiia et al. 2003, 170), una aproximación más apropiada para el accionista de la empresa y su libertad individual — siguiendo lo planteado por Milton Friedman (1970) - , pero menos apropiado a la responsabilidad fiduciaria de la organización. Se trata de un modelo en el que las empresas entregan recursos motivados por el deseo de "ser un buen ciudadano" sin esperar una retribución a cambio, y tienden a enfocarse en causas de alta relevancia social o pública, pero no vinculadas o afectadas por las operaciones de la empresa (Porter y Kramer 2002, 13).

Por otra parte, se ha reconocido que este tipo de acciones filantrópicas sirven como instrumento para fortalecer la legitimidad corporati- 
va y reputacional (Chen et al., 2008, 131), dando pie a un enfoque de legitimidad social, con un foco en la retribución de la opinión pública o de inversionistas interesados en determinadas acciones filantrópicas. Este enfoque busca apoyar causas favorecidas por los empleados, clientes o líderes de la comunidad, con un énfasis en asociar las contribuciones sociales como herramienta de relacionamiento y negociación con la comunidad, y en algunos casos con el fin de mitigar y anticipar efectos adversos de la instalación o de la operación de la empresa. Sin embargo, el énfasis en el foco reputacional o de licencia para operar sucede en forma generalmente reactiva. En la práctica, se concreta con una serie de acciones paliativas o defensivas de corto plazo, que responden a las demandas de cada stakeholder, o en causas de gran efecto comunicacional (el denominado cause-related marketing), en general relegadas a un encargado de la compañía que trabaja en forma separada de otras gerencias. Esto produce que el impacto social se difumine en múltiples acciones y se diluya la capacidad de fortalecer la competitividad de la empresa en el largo plazo, así como el impacto social de estas acciones. Este enfoque entiende la relación entre empresa y la sociedad como una tensión permanente que es necesario aplacar más que una mutua dependencia que deba fortalecerse (Porter y Kramer 2006, 4-5).

La diferencia entre los tres enfoques (altruista, reputacional o estratégico $)^{5}$ radica en cómo se conciben los aportes sociales y la responsabilidad social empresarial: como una acción individual del tomador de decisión a nivel organizacional (dueño o alta gerencia); como respuesta reactiva a demandas externas (ya sea para construir goodwill reputacional o licencia para operar); o bien como una estrategia interna de desarrollo sostenible del negocio. En este último enfoque, el diseño de los aportes sociales y su vínculo con la filantropía estratégica será única para cada empresa en base a sus valores internos y a las necesidades sociales que existan en el contexto competitivo de su operatividad (Smith 2003, 67; Porter y Kramer 2006, 5).

${ }^{5}$ El estudio del Cefis UAI señala que las empresas encuestadas que operan en Chile, respecto de sus aportes sociales, el 33 por ciento opera bajo un enfoque altruista; el 50 por ciento, bajo un enfoque de legitimidad social, y el 17 por ciento, bajo un enfoque estratégico (Aninat y Fuenzalida 2017, 46). 
Tabla 1. DIFERENCIAS PRINCIPALES EN LOS TIPOS DE ENFOQUE EN FILANTROPÍA CORPORATIVA

\begin{tabular}{|c|c|c|c|c|c|}
\hline $\begin{array}{l}\text { Tipo de } \\
\text { enfoque }\end{array}$ & $\begin{array}{l}\text { Concepción } \\
\text { de los apor- } \\
\text { tes sociales }\end{array}$ & $\begin{array}{l}\text { Definición } \\
\text { de ámbito } \\
\text { social al } \\
\text { cual des- } \\
\text { tinan los } \\
\text { aportes }\end{array}$ & $\begin{array}{l}\text { Principal } \\
\text { beneficiado }\end{array}$ & $\begin{array}{l}\text { Partici- } \\
\text { pación en } \\
\text { toma de } \\
\text { decisiones }\end{array}$ & $\begin{array}{l}\text { Importancia } \\
\text { del efecto } \\
\text { comunica- } \\
\text { cional de } \\
\text { los aportes } \\
\text { sociales }\end{array}$ \\
\hline Altruista & $\begin{array}{c}\text { Acción } \\
\text { individual del } \\
\text { tomador de } \\
\text { decisión }\end{array}$ & $\begin{array}{l}\text { Ámbitos de } \\
\text { relevancia } \\
\text { pública } \\
\text { o para la } \\
\text { sociedad }\end{array}$ & Sociedad & $\begin{array}{l}\text { Dueño o alta } \\
\text { gerencia }\end{array}$ & Baja \\
\hline $\begin{array}{l}\text { Legitimidad } \\
\text { social }\end{array}$ & $\begin{array}{c}\text { Reacción a } \\
\text { demandas } \\
\text { externas }\end{array}$ & $\begin{array}{l}\text { Ámbitos de } \\
\text { relevancia } \\
\text { para sus } \\
\text { stakeholders }\end{array}$ & $\begin{array}{l}\text { Posición } \\
\text { estratégica } \\
\text { empresa }\end{array}$ & $\begin{array}{l}\text { Gerencia de } \\
\text { relaciona- } \\
\text { miento con } \\
\text { stakeholders }\end{array}$ & Alta \\
\hline Estratégico & $\begin{array}{l}\text { Estrategia } \\
\text { interna para } \\
\text { la sosteni- } \\
\text { bilidad del } \\
\text { negocio }\end{array}$ & $\begin{array}{l}\text { Ámbitos de } \\
\text { relevancia } \\
\text { para la } \\
\text { sociedad y la } \\
\text { comunidad, } \\
\text { y el contexto } \\
\text { competitivo } \\
\text { de la } \\
\text { empresa }\end{array}$ & $\begin{array}{l}\text { Posición } \\
\text { estratégica } \\
\text { empresa + } \\
\text { sociedad }\end{array}$ & $\begin{array}{c}\text { Participación } \\
\text { de varias } \\
\text { gerencias }\end{array}$ & Indiferente \\
\hline
\end{tabular}

Fuente: Elaboración de las autoras.

\section{HIPÓTESIS}

Como se señaló en la introducción, este artículo busca aportar a los vacíos identificados por la literatura en términos de modelos de implementación de una filantropía estratégica (Thorne y Ferrell 2000, 703). Para este fin, evalúa la existencia de relaciones entre el análisis del contexto competitivo en la toma de decisiones de los aportes sociales, por una parte, y la implementación de un enfoque de filantropía estratégica, por la otra.

De esta manera, es relevante distinguir que la filantropía estratégica no consiste sólo en implementar una estrategia en los aportes sociales, ordenada en los procedimientos y métodos para entregar donaciones. Más bien, la filantropía estratégica indica que los recursos corporativos que se donan tienen sentido e impacto en la firma, así como en la comunidad que recibe esos recursos (Post y Waddock 1995 
en Saiia et al. 2003, 185-186). En base a lo dicho, adoptar un enfoque estratégico requiere implementar sistemas a nivel de gestión en la organización, que incorporen el profesionalismo en la función de donar con el mismo rigor que otras funciones de la empresa, al tiempo que un vínculo entre la identidad de la compañía y su filantropía (Maas y Liket 2010, 447). Diversos autores ${ }^{6}$ han identificado una serie de factores que permiten implementar o maximizar una filantropía estratégica:

- el involucramiento de varias gerencias de primera línea en la definición de los aportes sociales de la empresa;

- la necesidad de incorporar sistemas y criterios, que permitan seleccionar a los mejores destinatarios;

- acciones de señalización para abrir pasos a otros donantes, que permitan sumar apoyos mutuos para una misma institución beneficiaria o generar esfuerzos colectivos a un mismo ámbito;

- la relevancia de colaborar con las entidades donatarias, para mejorar su desempeño y fortalecimiento institucional;

- establecer alianzas de mediano o largo plazo, que entreguen el tiempo necesario para lograr un mejoramiento significativo de las organizaciones donatarias;

- la relevancia de fortalecer el conocimiento y la práctica;

- la promoción de la innovación social;

- la medición de resultados del valor social generado.

Sin embargo, un factor anterior a la implementación de la filantropía estratégica es la definición de una estrategia interna con una visión de sostenibilidad del negocio. Elemento clave es el análisis del contexto competitivo para definir hacia cuáles necesidades sociales enfocará la empresa sus acciones filantrópicas. Una visión completa del entorno facilita la adopción de un enfoque estratégico.

En el ámbito competitivo, Porter $(1990,78)$ distingue una serie de elementos interrelacionados necesarios para un ambiente favorable a la productividad de negocios. Ellos son:

- condiciones factoriales o insumos necesarios para la producción, vinculados a la educación en ámbitos que tienen relación con la compañía, a la calidad de vida de los territorios donde opera, al manejo sus-

6 Varios de estos elementos son identificados por Porter y Kramer (1999; 2002, 9-11) que denominan "maximizadores de la filantropía estratégica". 
tentable de los recursos naturales relacionados con su forma de operar y al conocimiento técnico o científico relativo al negocio (en suma, los recursos humanos y naturales);

- condiciones de demanda (valorización de productos y sofisticación de clientes y estándares); esto es, lo vinculado al conocimiento, valorización, calidad y estándares de los servicios y productos de la compañía, junto con la educación sobre el consumo responsable de sus productos o servicios;

- contexto para la estrategia y competencia (regulaciones, competitividad, propiedad intelectual), que se relacionan con la regulación e incentivos de libre competencia (transparencia, propiedad intelectual, reducción de corrupción, antimonopolio, etcétera);

- industrias relacionadas y de soporte (proveedores y desarrollo de clusters); esto es, lo vinculado al desarrollo de la economía local y de proveedores locales en zonas donde opera la compañía.

La debilidad, en cualquier parte de este contexto, puede erosionar la competitividad de una región o país como ubicación para negocios (Porter y Kramer 2002, 5). El valor del análisis del contexto competitivo se erige, entonces, como factor esencial para adoptar un enfoque estratégico de aportes sociales. Sin embargo, la clave es reconocer que, en el ámbito de sus aportes sociales, las empresas no pueden abordar cada área del contexto competitivo, por lo cual se hace necesario identificar las áreas sociales con mayor valor estratégico, esto es, con mayor potencial para generar valor para la sociedad y para su propia competitividad (Porter y Kramer, 2002, 12; 2006, 9). Es por eso que la forma de implementación requiere el desarrollo de un proceso de análisis de oportunidades en el contexto competitivo de la empresa, y, en este escenario, la participación de las distintas gerencias y del gobierno corporativo cobra especial relevancia (Aninat y Fuenzalida 2017, 45) y facilita luego la implementación de un enfoque estratégico en sus distintos elementos.

En este marco, este artículo busca comprender si existe una relación entre el análisis del contexto competitivo con los distintos elementos que permiten implementar la filantropía estratégica.

Las hipótesis de trabajo son las siguientes:

i) Hipótesis 1 (implementación): Las empresas que llevan a cabo un análisis del contexto competitivo para la toma de decisiones de los 
aportes sociales adoptan un mayor nivel de estrategia en los procedimientos para la implementación de estos aportes.

En segundo término, y tal como se señaló anteriormente, indagamos también en la relación entre análisis del contexto competitivo para los aportes sociales y la valorización de las empresas bajo una óptica de mercado, aportando nuevos argumentos para desarrollar una relación integrada entre empresa y sociedad. Este enfoque win-win en las contribuciones sociales requiere demostrar el valor generado para la empresa. Si bien se ha identificado la generación de valor en términos de atracción y aseguramiento de recursos necesarios para la productividad (talento humano, inversionistas, capital moral entre stakeholders, reduciendo conflictos y controversias, capacidad de innovación), su cuantificación ha sido un desafío en la investigación académica. Por ello es significativo el aporte del estudio de Eccles et al. (2011), en el cual analizaron los resultados de rentabilidad en 18 años de un grupo de 180 empresas en Estados Unidos, diferenciando entre empresas con un alto nivel de sostenibilidad y empresas con un bajo nivel de sostenibilidad. En Eccles et al. se demuestra que las empresas con alto nivel de sostenibilidad logran un desempeño financiero más alto - mayor valor accionario y mayor retorno sobre la inversión medido en return-on-equity (ROE) y return-on-assets (ROA) — que las con bajo nivel de sostenibilidad, dando cuenta de que la integración temprana de políticas de sostenibilidad en el modelo de negocio y su estrategia puede ser una fuente de ventaja competitiva en el largo plazo (Eccles et al. 2011, 2835-2836). En esta misma línea, el estudio de Wang et al. — desarrollado en las principales empresas donantes en Estados Unidos - logra mostrar que, en la medida en que la filantropía ayuda a asegurar recursos críticos de varios stakeholders y reduce los riesgos de exposición de las compañías, se genera en el tiempo retorno financiero (Wang et al. 2008, 144). Dado que entendemos la filantropía corporativa como un elemento discrecional y voluntario que corona la práctica de responsabilidad social empresarial (hoy contenida en el concepto de sostenibilidad empresarial), buscamos analizar si existe también esta relación entre un análisis del contexto competitivo en los aportes sociales, por un lado, y mayor valoración de mercado de las empresas, por el otro.

ii) Hipótesis 2 (valorización): Existe una relación positiva entre el análisis del contexto competitivo para la toma de decisiones de los aportes sociales y la valorización de mercado de las empresas. 
Con estos elementos, nuestro artículo intenta evaluar la relevancia que tiene la realización de un análisis del contexto competitivo en la toma de decisiones de los aportes sociales, cuando se busca adoptar un enfoque estratégico, que genere valor para la empresa y para la sociedad.

\section{METODOLOGÍA}

Para comprobar las hipótesis, se toma como unidad de análisis las sociedades anónimas abiertas inscritas en el Registro de Valores de la Superintendencia de Valores y Seguros en Chile. Se acotó la muestra a un solo país (Chile) para no introducir variables del contexto normativo, competitivo y cultural en las unidades observadas. Se definió analizar este tipo de empresas debido al alto componente de business exposure, es decir, su amplia apertura y grado de vulnerabilidad hacia su entorno social. Lo anterior deriva del hecho de estar bajo escrutinio por un amplio rango de grupos de interés o stakeholders -incluyendo a los accionistas minoritarios - que manifiestan expectativas sobre la conducción y el comportamiento de las firmas. En este tipo de empresas, los aportes sociales de la compañía se entienden como una respuesta propia de la interpretación que los directivos hacen de la exposición de la compañía en el ámbito institucional y a la comunidad en general (Saiia et al. 2003, 175).

\subsection{Muestra}

Para la identificación de la muestra, se utilizaron los siguientes criterios de inclusión:

- Empresas donantes. Según registros del Servicio de Impuestos Internos, solicitados de acuerdo a la Ley 20.285 Sobre Acceso a la Información Pública, se identificaron a las empresas que habían realizado donaciones sociales acogidas en la legislación chilena entre los años 2013 y 2015.

- Empresas que publicaron en sus memorias o reportes de sustentabilidad, entre los años 2013 y 2015, alguna acción vinculada a la inversión social o contribuciones sociales. Al efecto, se realizó una revisión de tales documentos para todas las empresas nacionales, abiertas a la Bolsa de Comercio registradas en la Superintendencia de Valores y 
Seguros (SVS), utilizando los siguientes criterios de búsqueda: "dona", "donación(es)", "aporte(s) social(es)", "ayuda social", "apoyo", "comunidad", "fundación”, siempre y cuando hicieran referencia a alguna donación o contribución social concreta.

El resultado del proceso permitió identificar un universo de estudio de 129 empresas que cumplían con algunos de los dos criterios presentados. Entre ellas, el 84 por ciento contaba con registros de donación acogidas a la legislación chilena entre el 2013 y 2015, mientras que el 67 por ciento declaraba algún tipo de acción relativa a donaciones o contribuciones sociales en sus memorias o reportes de sustentabilidad en el mismo horizonte temporal.

\subsection{Instrumento}

En base a la revisión literaria se elaboró una encuesta que fue estructurada para evaluar el enfoque subyacente a las donaciones y contribuciones sociales que realizan las empresas, la relevancia del análisis del contexto competitivo en la definición de aportes sociales y recolección de información respecto de la presencia de factores que permiten efectuar o maximizar la implementación de una filantropía estratégica (destinados al análisis de la hipótesis 1, implementación).

El cuestionario construido constó de 39 preguntas, con categorías de respuesta principalmente en escala Likert, además de algunas preguntas abiertas sobre percepciones. El instrumento fue testeado en actores vinculados al mundo empresarial y sustentabilidad, de manera de validar el uso del vocabulario y el tiempo de aplicación.

El diseño muestral fue de carácter censal (dada la finitud y tamaño del universo) y su aplicación fue realizada vía online, entre los meses de septiembre y diciembre de 2015. Se logró la participación efectiva de 54 firmas (42 por ciento del universo identificado), en su mayoría empresas grandes de diversos sectores económicos (72 por ciento de las cuales clasifican en el rango superior de facturación del SII, y 82 por ciento de las cuales cuenta con más de 200 empleados).

La encuesta fue contestada por un representante de la empresa designado por la misma institución, pero se exigió que estuviera a cargo de la toma de decisiones sobre donaciones o contribuciones sociales. La encuesta fue respondida por: el gerente general (18 por ciento); el 
gerente o encargado del área de sostenibilidad o responsabilidad social empresarial (50 por ciento); gerente o encargado de asuntos públicos, marketing o comunicaciones (22 por ciento); gerente o encargado de administración y finanzas (6 por ciento), y gerente o encargado de recursos humanos (4 por ciento).

Considerando que el instrumento de recolección de información fue autorreportado y que la participación de las empresas fue voluntaria, es posible que exista un sesgo en los resultados asociado a las características específicas de las empresas que accedieron a participar.

\subsection{Escala de análisis}

Para el análisis de las hipótesis se utilizó como variable principal una escala de estrategia en el estudio del contexto competitivo para la toma de decisiones de los aportes sociales. Esta escala fue construida a partir de 10 preguntas del cuestionario, en las que se evaluó el nivel de relevancia con que fueron consideradas al momento de definir el foco de los aportes sociales y los elementos interrelacionados necesarios para un ambiente favorable a la productividad de negocios. Estos elementos, definidos como interrelacionados y necesarios en base a Porter (Porter 1990, 78; Porter y Kramer 2006, 9), se presentan a continuación:

- Educación y capacitación en ámbitos que tienen relación con la compañía.

- Calidad de vida de los territorios donde operan y viven los trabajadores de la compañía.

- Manejo sustentable de los recursos naturales relacionados con la compañía.

- Conocimiento técnico o investigación científica relacionado con la compañía.

- Conocimiento y valorización de los productos o servicios de la compañía.

- Educación sobre el consumo responsable de los productos o servicios de la compañía.

- Calidad y estándares de los productos o servicios de la compañía.

- Regulación e incentivos de libre competencia (transparencia, propiedad intelectual, reducción de corrupción, antimonopolio, etcétera).

- Economía local en zonas donde opera la compañía.

- Desarrollo de proveedores locales. 
Las preguntas tuvieron como posibles respuestas una escala Likert del 1 al 4, a partir de las cuales se construyó una escala mediante análisis factorial exploratorio, y validado con análisis de fiabilidad utilizando alpha de Cronbach de 0,898, ${ }^{7}$ con 10 elementos. Este resultado permitió una reducción de los 10 elementos a una sola dimensión, por lo que cada una de las preguntas tiene un peso equivalente.

Los resultados entregaron una escala cuyos valores posibles se encontraban en el rango del 1 al 4, en el que 1 representa el nivel más bajo de incorporación de elementos de la cadena de valor y contexto competitivo, y 4 el nivel más alto. Posteriormente esta escala fue estandarizada, por lo que los resultados presentados muestran los valores de esta escala con media 0 y desviación estándar 1 . La escala estandarizada, de varianza 1 y media 0 , toma la forma similar a la normal, con asimetría levemente negativa y curtosis levemente positiva. ${ }^{8}$

Esta escala fue sometida a test de diferencia de medias, para muestras independientes y para cada uno de los análisis requeridos en las dos hipótesis. La escala fue calculada para 48 de los 54 casos que respondieron la encuesta, ya que se excluyeron seis empresas que no contestaron al menos una de las 10 preguntas que la componen.

\subsection{Información secundaria}

Para la hipótesis 2 (valorización) se realizó un análisis de información secundaria, con el objetivo de revisar la participación de las empresas en un índice confiable y público, indicativo de la valorización de mercado de las empresas, que permitiera su análisis frente a la escala de estrategia en el de contexto competitivo para la toma de decisiones que hacen los aportes sociales.

En base a juicio de experto, se seleccionó como indicador la presencia en el Índice de Precios Selectivo de Acciones (IPSA), elaborado por la Bolsa de Comercio de Santiago, reconocido como el principal índice bursátil de Chile e indicador de rentabilidad en términos de valorización accionaria de mercado. Dados que su finalidad es valorar

${ }^{7}$ Alpha de Cronbach sobre 0,7 es considerado altamente confiable y consistente.

${ }^{8}$ Para más detalles de los momentos de la distribución, contactarse con alguna de las autoras. 
las variaciones de precio de los títulos con mayor liquidez o presencia en el mercado y que su elaboración es sobre un número restringido (40 empresas, cuya base se renueva anualmente), este índice permitía para los fines del estudio contar con una base de comparación objetiva y externa al estudio mismo, pero atingente a la unidad de análisis. Para este estudio se identificó a las empresas del universo con presencia en este índice durante el año 2015.

\section{RESULTADOS}

Tal como se ha señalado, el artículo plantea el análisis del contexto competitivo como factor necesario para establecer una filantropía estratégica, considerando el enfoque estratégico como aquel que facilita que los aportes sociales de las empresas generen mayor valor para la sociedad y para la empresa. Por ello, en primer lugar, proponemos estudiar la relación entre el análisis del contexto competitivo para los aportes sociales con factores que permiten su implementación estratégica, y, en segundo lugar, la relación entre este uso con un indicador de valorización económica de las empresas.

Entonces:

\subsection{Hipótesis 1 (implementación)}

Las empresas que llevan a cabo un análisis del contexto competitivo para la toma de decisiones de los aportes sociales cuentan con un mayor nivel de estrategia en los procedimientos para la implementación de los mismos.

La literatura de filantropía estratégica señala ciertos factores en la implementación de los aportes sociales, los cuales son relevantes en la medida en que permiten maximizar el aporte al desarrollo social de las contribuciones empresariales. Estos factores - que se describen a continuación - se abordaron en la encuesta a las empresas y se analizaron utilizando la escala de análisis competitivo para los aportes sociales, con el fin de dar respuesta a la hipótesis 1 (implementación).

La literatura señala la relevancia de la participación de la alta gerencia de la empresa en el enfoque estratégico. Eccles et al. señalan que en aquellas empresas que consideran los objetivos sociales y medioambientales como centrales para su estrategia y operaciones, el 
directorio es más propenso a tener responsabilidad directa sobre estos temas $(2011,2838)$. Saiia et al. señalan que cuando la alta gerencia inicia la integración de los aportes sociales en la orientación general de la compañía, sólo entonces, los aportes sociales dejan de ser funcionales y se crea la posibilidad de que una estrategia filantrópica se convierta efectivamente en una filantropía estratégica (2003, 182). En suma, el énfasis que este enfoque tiene en focalizar la filantropía corporativa en el contexto competitivo de la empresa requiere que la toma de decisiones no quede delegada en las gerencias orientadas a comunicaciones o atención a stakeholders (por ejemplo, asuntos públicos). Más bien, es necesario integrar a las distintas gerencias - que tienen la capacidad de identificar áreas relevantes para focalizar estos recursos-, en un proceso liderado por el gerente general y orientado a desarrollar una estrategia focalizada en mejorar el contexto de la compañía (Porter y Kramer 2002, 12).

En línea con lo señalado, los resultados de la encuesta, sometidos a la escala de análisis del contexto competitivo para los aportes sociales, permiten identificar que las empresas donde participan tres o más gerencias en la toma de decisiones sobre el foco de las donaciones obtienen un puntaje mayor en la escala, en comparación con aquellas empresas en que participan dos o menos. Esta diferencia es estadísticamente significativa al 95 por ciento de confianza.

Tabla 2. PARTICIPACIÓN DE DISTINTAS GERENCIAS DE LA EMPRESA EN LA TOMA DE DECISIONES DE LOS APORTES SOCIALES

\begin{tabular}{lccc}
\hline & \multicolumn{3}{c}{ Participación de gerencias de la empresa } \\
\hline & 3 o más & 2 o menos & Diferencia \\
\hline Promedio escala de análisis del & 0,26 & $-0,29$ & $0,56^{\star *}$ \\
contexto competitivo & $(0,75)$ & $(1,15)$ & \\
\hline
\end{tabular}

$N=48 .{ }^{*} p<0,10 * * 0<0,05{ }^{* * *} p<0,01$.

Errores estándares robustos entre paréntesis.

Valores estandarizados en escala del contexto competitivo.

De esta forma comprobamos que en las empresas donde hay una participación de un mayor número de gerencias en la toma de decisiones sobre los aportes sociales se consideran más elementos en la toma de decisiones sobre el foco de sus aportes sociales. 
Los autores Porter y Kramer (1999; 2002, 9-11) definen una serie de factores específicos que permiten maximizar el valor social que generan los aportes filantrópicos corporativos. Estos elementos, denominados por los autores como maximizadores de filantropía estratégica, incluyen, en primer término, la necesidad de incorporar sistemas y criterios que permitan seleccionar a los mejores destinatarios (lo que requiere diferenciar entre organizaciones beneficiarias de alto conocimiento público de aquéllas con alta capacidad de gestión que logran mayor impacto social). Los autores señalan también la necesidad de desarrollar acciones de señalización para abrir pasos a otros donantes, las cuales permitan sumar apoyos mutuos para una misma institución beneficiaria o generar esfuerzos colectivos en un mismo ámbito, mitigando el problema del free rider y maximizando resultados sociales. Asimismo, los autores destacan la relevancia de colaborar con las entidades donatarias para mejorar su desempeño, de manera de lograr que sean más efectivas, a través de un apoyo dirigido a lograr su fortalecimiento institucional y, también, mediante alianzas de mediano o largo plazo, que entreguen el tiempo necesario para lograr un significativo mejoramiento de las organizaciones donatarias, elemento señalado también por Austin et al. bajo la denominación de estrategia integrativa $(2005,5)$.

Junto con lo anterior, Porter y Kramer (1999; 2002, 11-12) identifican la importancia de fortalecer el conocimiento y la práctica. La innovación, argumentan, conduce a la productividad en el sector sin fines de lucro tanto como en el sector comercial. Promover, entonces, el desarrollo del conocimiento y la innovación requiere que las empresas que buscan maximizar su filantropía estratégica realicen acciones para impulsar la investigación, la transferencia y la difusión de conocimiento de innovación social en temáticas relacionadas con sus aportes sociales.

Un último elemento maximizador del valor generado por los aportes sociales corporativos es la medición de resultados respecto de los efectos generados por éstos (Porter y Kramer 2002, 13). Al medir los resultados de impacto social de los aportes filantrópicos para la toma de decisiones, se evita desperdiciar recursos, se maximizan los beneficios y se evitan posibles costos en la sociedad. Para ello, es necesario recopilar y analizar datos relacionados con el impacto social, así como el efecto reputacional en distintos stakeholders (Maas y Liket 2010, 446).

Al utilizar la escala de análisis competitivo para los aportes sociales y evaluar su comportamiento (promedio) respecto de los factores de 
implementación o maximizadores de filantropía estratégica recogidos en la encuesta a las empresas que operan en Chile, es posible comprobar lo siguiente:

Las empresas chilenas que, para seleccionar a la institución beneficiaria, utilizan criterios estratégicos —es decir, consideran elementos vinculados a la capacidad de gestión o experiencia (eficiencia administrativa o resultados anteriores logrados) de la entidad beneficiariaobtienen un puntaje significativamente mayor conforme a la escala de estrategia en el análisis del contexto competitivo, respecto de las empresas que utilizan otros criterios asociados a legitimidad social (visibilidad y capacidad comunicacional) o a altruismo (filosofía o misión social de la entidad beneficiaria o, bien, conocimiento o relación con los directivos de la entidad beneficiaria).

Tabla 3. CRITERIOS DE SELECCIÓN DE ENTIDADES BENEFICIARIAS POR PARTE DE LAS EMPRESAS

\begin{tabular}{lccc}
\hline & Criterios estratégicos & Otros criterios & Diferencia \\
\hline Promedio escala de análisis & 0,05 & $-0,38$ & 0,43 \\
del contexto competitivo & $(0,96)$ & $(1,27)$ & \\
\hline
\end{tabular}

$\mathrm{N}=48 .{ }^{*} \mathrm{p}<0,10$ ** $\mathrm{p}<0,05{ }^{* * *} \mathrm{p}<0,01$.

Errores estándares robustos entre paréntesis.

Valores estandarizados en escala del contexto competitivo.

Con esto se cumple que, en términos de tendencia, las empresas que utilizan criterios que permiten seleccionar a lo que se entiende como mejores destinatarios toman también en cuenta mayores elementos del contexto competitivo, al momento de la toma de decisiones sobre el foco de sus aportes sociales.

Por otra parte, las empresas que han promovido la participación de otras empresas, ya sea del mismo o de otro sector, obtienen un puntaje mayor en la escala respecto a las que no lo han hecho. Esta diferencia es significativa al 90 por ciento de confianza.

Así, comprobamos que las empresas que han realizado acciones de señalización para sumar a otras compañías en el apoyo de instituciones beneficiarias consideran mayores elementos del contexto competitivo en la toma de decisiones sobre el foco de sus aportes sociales. 
Tabla 4. PROMOCIÓN DE LA PARTICIPACIÓN DE OTRAS EMPRESAS EN APOYO A UNA INSTITUCIÓN BENEFICIARIA

\begin{tabular}{lccc}
\hline & \multicolumn{3}{c}{$\begin{array}{c}\text { Empresas que han incentivado o convocado a } \\
\text { otras empresas a sumar apoyos a una institución } \\
\text { beneficiaria }\end{array}$} \\
\hline & $\mathrm{Si}$ & No & Diferencia \\
\hline $\begin{array}{l}\text { Promedio escala de análisis del } \\
\text { contexto competitivo }\end{array}$ & 0,53 & $-0,10$ & $0,64^{*}$ \\
\hline
\end{tabular}

$\mathrm{N}=48 .{ }^{*} \mathrm{p}<0,10$ ** $\mathrm{p}<0,05^{* * *} \mathrm{p}<0,01$.

Errores estándares robustos entre paréntesis.

Valores estandarizados en escala del contexto competitivo.

En otro aspecto, se analizó también el comportamiento de empresas que han establecido una alianza con una institución beneficiaria a mediano plazo (por al menos dos años), en cuyo marco se han realizado acciones que ayudan a mejorar su desempeño en áreas como el fortalecimiento institucional, el intercambio de conocimientos o el equipo profesional conjunto para el desarrollo de iniciativas. Las empresas que, al responder, dijeron haber establecido este tipo de alianzas cuentan con un promedio en la escala de análisis del contexto competitivo estadísticamente mayor que aquellas que no cuentan con este tipo de alianzas. Esta diferencia es significativa al 99 por ciento de confianza.

Tabla 5. EMPRESAS QUE HAN ESTABLECIDO ALIANZAS CON INSTITUCIONES BENEFICIARIAS

\begin{tabular}{lccc}
\hline & \multicolumn{3}{c}{$\begin{array}{c}\text { Empresas que han establecido alianza } \\
\text { con instituciones beneficiarias }\end{array}$} \\
\hline & $\mathrm{Si}$ & No & Diferencia \\
\hline Promedio escala de análisis del & 0,38 & $-0,85$ & $1,24^{* * *}$ \\
contexto competitivo & $(0,7)$ & $(1,11)$ & \\
\hline
\end{tabular}

$\mathrm{N}=48 .{ }^{*} \mathrm{p}<0,10{ }^{* *} \mathrm{p}<0,05^{* * *} \mathrm{p}<0,01$.

Errores estándares robustos entre paréntesis.

Valores estandarizados en escala del contexto competitivo.

Con esto se cumple la tesis según la cual las empresas que colaboran y se comprometen a mejorar el desempeño de las instituciones beneficiarias han tomado en cuenta mayores elementos del contexto competitivo en la toma de decisiones sobre el foco de sus aportes sociales. 
Respecto a las empresas que han incentivado iniciativas sociales innovadoras a través de reconocimientos o mentoría, hay que señalar que ellas obtienen un puntaje mayor en la escala de estrategia en el análisis del contexto competitivo. Esta diferencia es significativa al 95 por ciento de confianza.

Tabla 6. INCENTIVOS A LA INNOVACIÓN SOCIAL

\begin{tabular}{lccc}
\hline & \multicolumn{3}{c}{$\begin{array}{c}\text { Empresas que han entregado premios, } \\
\text { reconocimiento o mentoria a emprendimientos } \\
\text { sociales innovadores }\end{array}$} \\
\hline Pi & $\mathrm{Si}$ & Diferencia \\
\hline contexto competitivo & 0,45 & $-0,19$ & $0,64^{* *}$ \\
\hline
\end{tabular}

$\mathrm{N}=48 .{ }^{*} \mathrm{p}<0,10$ ** $\mathrm{p}<0,05^{* * *} \mathrm{p}<0,01$.

Errores estándares robustos entre paréntesis.

Valores estandarizados en escala del contexto competitivo.

De esta forma, se observa que las empresas que promueven la innovación social a través de su desarrollo o reconocimiento, efectivamente, han tomado en cuenta mayores elementos del contexto competitivo en la toma de decisiones sobre el foco de sus aportes sociales.

Por último, las empresas que han evaluado los resultados de sus aportes sociales - ya sea a nivel de los beneficios sociales logrados directamente por los proyectos financiados, de los resultados comunicacionales (en prensa u otros canales) o de los resultados obtenidos en algún stakeholder de la empresa - obtienen un puntaje mayor en la escala de estrategia en el análisis del contexto competitivo. Esta diferencia es significativa al 95 por ciento de confianza.

En suma, es posible establecer la existencia de la hipótesis 1 (implementación), según la cual las empresas que realizan análisis de su contexto competitivo en la toma de decisiones sobre sus aportes sociales muestran un mayor nivel de estrategia en prácticamente todos los factores analizados: participación de mayor número de altos directivos de la empresa; selección de los mejores destinatarios; acciones de señalización para abrir paso a otros donantes; colaboración para mejorar el desempeño de la entidad donataria, y promoción del desarrollo de la innovación social, todos ellos factores relevantes del enfoque estratégico 
en los aportes sociales. Las diferencias observadas son estadísticamente significativas, al menos al 90 por ciento de confianza. Asimismo, el análisis sobre la selección de los mejores destinatarios también muestra una diferencia relevante a nivel de tendencias.

Tabla 7. MEDICIÓN DE RESULTADOS DE APORTES SOCIALES

\begin{tabular}{lccc}
\hline & \multicolumn{3}{c}{$\begin{array}{c}\text { Empresas que han medido resultados } \\
\text { de los aportes sociales }\end{array}$} \\
\hline & $\mathrm{Si}$ & No & Diferencia \\
\hline $\begin{array}{l}\text { Promedio escala de análisis del } \\
\text { contexto competitivo }\end{array}$ & 0,24 & $-0,40$ & $0,64^{* *}$ \\
\hline
\end{tabular}

$N=48 .{ }^{*} p<0,10 *{ }^{* *} p<0,05{ }^{* * *} p<0,01$.

Errores estándares robustos entre paréntesis.

Valores estandarizados en escala del contexto competitivo.

\subsection{Hipótesis 2 (valorización)}

Existe una relación positiva entre el análisis del contexto competitivo para la toma de decisiones de los aportes sociales y la valorización de mercado de las empresas.

Como se mencionara anteriormente, estudios internacionales han demostrado los efectos en rentabilidad que genera en el mediano y largo plazo la incorporación de altos niveles de sostenibilidad. Eccles et al. (2011, 2849 -2850) muestran que, en empresas abiertas a bolsa en Estados Unidos, aquéllas con altos indicadores de sostenibilidad logran en el tiempo una rentabilidad bursátil acumulada significativamente mayor en comparación con empresas con baja sostenibilidad.

La sostenibilidad es un concepto que se enmarca o sobrepone al de responsabilidad social empresarial, y la filantropía corporativa es el elemento más discrecional y voluntario que corona la responsabilidad social. En este ámbito, Wang et al. comprueban que la filantropía corporativa mejora la performance financiera de las empresas al permitirles mayor control sobre recursos de sus stakeholders (Wang et al. 2008, 156). En este marco, es posible plantear que existe una relación positiva entre un enfoque estratégico (vía análisis del contexto competitivo en los aportes sociales) y la valoración bursátil de las compañías. 
La hipótesis 2 (valorización) se investiga desde un indicador objetivo, público y externo al estudio, indicativo de la valorización de mercado de las empresas, cual es la presencia de las empresas donantes en el IPSA de la Bolsa de Santiago durante el año 2015, examinado en base a su relación en la escala de análisis del contexto competitivo para sus aportes sociales. Las empresas IPSA tienen un puntaje mayor en esta escala en comparación con aquellas que no están presentes en este índice.

Tabla 8. PARTICIPACIÓN DE LAS EMPRESAS ANALIZADAS EN EL ÍNDICE IPSA

\begin{tabular}{lccc}
\hline & \multicolumn{3}{c}{ Presencia en el IPSA } \\
\hline Si & No & Diferencia \\
\hline $\begin{array}{l}\text { Promedio escala de análisis del } \\
\text { contexto competitivo }\end{array}$ & 0,21 & $-0,08$ & 0,29 \\
\hline
\end{tabular}

$\mathrm{N}=48 .{ }^{*} \mathrm{p}<0,10{ }^{* *} \mathrm{p}<0,05$

Errores estándares robustos entre paréntesis.

Valores estandarizados en escala del contexto competitivo.

Así, en términos de tendencia, se cumple la hipótesis 2 (valorización), ya que se observa que las empresas de mayor valorización de mercado — según participación en el IPSA - han tomado en cuenta más elementos del contexto competitivo en la toma de decisiones sobre sus aportes sociales.

\section{DISCUSIÓN Y CONCLUSIONES}

El enfoque estratégico se ha presentado como aquel que permite maximizar el valor que los aportes sociales de las empresas generan a la sociedad y que, al mismo tiempo, fortalece la estrategia competitiva del negocio. Bajo este doble objetivo, se justifica su desarrollo ante el juicio de los diferentes tipos de shareholders (controladores y minoritarios). Asimismo, el enfoque estratégico permite a las empresas desarrollar un sistema de aportes sociales corporativos dentro de un contexto en el que se les exige mayor productividad, se les aumentan las exigencias regulatorias y en el que la expectativa pública exige mayor coherencia entre las acciones de responsabilidad social y las estrategias productivas del negocio. De hecho, si estos aportes son guiados 
por los valores centrales y perdurables de la empresa serán genuinos y con mayor propensión a ser percibidos como tales, lo que permitirá a la empresa proyectar una "consistencia identitaria" ante un escenario de múltiples stakeholders (Godfrey 2005, 795).

Considerando que las empresas son responsables del 84 por ciento de las donaciones registradas en Chile, es importante que las contribuciones sociales corporativas generen un valor destacado para la sociedad. Esto es especialmente relevante cuando el sistema de incentivos tributarios a las donaciones establece reducciones significativas al costo de la donación para los contribuyentes de primera categoría, estableciendo un cofinanciamiento indirecto por parte del Estado y un costo fiscal asociado a las donaciones privadas. ${ }^{9}$

El análisis de este estudio, en torno a los componentes del enfoque estratégico en los aportes sociales corporativos, busca poner en discusión una agenda que promueva un alineamiento sinérgico al enfocar recursos corporativos a problemas sociales o a temáticas que resuenan con los valores claves y la misión de la empresa y, con ello, generen una mirada de largo plazo y de resultados sociales más relevantes para la sociedad.

El enfoque estratégico está basado en reconocer que los recursos que la empresa dispone son financieros, pero también de know how y redes. Bajo esta perspectiva, se maximiza el valor de los aportes sociales cuando el foco elegido tiene que ver con las competencias propias de la empresa y con un ámbito de convergencia de intereses entre aquello relevante, beneficioso para la sociedad, y aquello necesario para la compañía, logrando un compromiso de largo aliento que facilita lograr un impacto social significativo (Porter y Kramer 2002, 3).

Considerando los vacíos que existen en torno a los componentes de un enfoque estratégico, este artículo contribuye a identificar importantes elementos para la adopción de un enfoque estratégico, al indagar en las relaciones entre el análisis del contexto competitivo y la adopción e implementación de elementos propios del enfoque estratégico. Los resultados demuestran que existe una relación positiva entre el análisis del con-

${ }^{9}$ Cabe recordar que existen más de 50 cuerpos legales y normativas que regulan las donaciones en Chile y, según la legislación que utilice el donante, puede obtener un crédito tributario por hasta el 50 por ciento de la donación y acoger el 50 por ciento restante a ser reconocido como gasto o, simplemente, llevarlo a gasto. Siempre con un tope general del 5 por ciento de la renta líquida imponible. 
texto competitivo y la adopción e implementación de elementos propios del enfoque estratégico. Se concluye, entonces, que el análisis del contexto competitivo podría determinar la adopción de un enfoque estratégico, lo que debiera ser confirmado en futuras investigaciones de causalidad.

Asimismo, el artículo identifica también una relación positiva entre el análisis del contexto competitivo en los aportes sociales, por una parte, $\mathrm{y}$ un indicador de valorización bursátil de las empresas, por la otra. De esta forma, manifiesta la relevancia del análisis del contexto competitivo para adoptar un enfoque estratégico en los aportes sociales y, con ello, beneficiar, a la vez, tanto a la sociedad como a la empresa.

El estudio presenta ciertas limitaciones. Uno de los instrumentos de recolección de la información (la encuesta a empresas) es autorreportado y su participación fue voluntaria, por lo que es posible que exista un sesgo en las respuestas o en las características específicas de las empresas que participaron. Por otro lado, el análisis se vería enriquecido si se contara con mayores indicadores para medir niveles de rentabilidad financiera de las empresas y así observar su correlación con el análisis del contexto competitivo.

Asimismo, sería deseable explorar posibles relaciones de causalidad entre las variables implicadas en este estudio, para lo cual sería necesario recolectar variables de control que permitan comprender la dirección de esta relación, como también indagar en la existencia de otras variables que estén correlacionadas con el análisis del contexto competitivo, identificables como variables omitidas en el análisis causal multivariado.

Con todo, este artículo corresponde a un primer esfuerzo en esa dirección, considerando la escasez de datos públicos sistematizados e integrados respecto de los donantes en Chile y de la práctica de aportes sociales de parte del sector empresarial.

Materia de futuras investigaciones será indagar en la diferencia de resultados de impacto social que logran las empresas con enfoque estratégico respecto de aquellas que adoptan otro tipo de enfoques. Asimismo, es de interés estudiar el nivel de integración de los aportes sociales en las estrategias de sostenibilidad de las empresas, y los efectos en otras variables relacionadas con la valorización de distintos tipos de empresas (por ejemplo, aquéllas de alta relación con comunidades versus proveedoras generales de servicios). 
Finalmente, se hace necesario estudiar una posible diferenciación de las políticas de incentivos tributarios a las donaciones sociales de empresas que permita impulsar, en este tipo de donante, factores relacionados con la implementación de la filantropía estratégica, en especial aquellas que fomentan la innovación social, las alianzas de largo plazo y los estudios de impacto social. De esta forma, se pueden establecer políticas que permitan incentivar la adopción de un enfoque que ayude a asegurar mayor valor para la sociedad y para las empresas.

\section{REFERENCIAS}

Aninat, Magdalena \& Isidora Fuenzalida. 2017. Estrategia en los aportes sociales de las empresas. Santiago: Centro de Filantropía e Inversiones Sociales UAI.

Austin, J, Reficco \& Equipo de investigación SEKN. 2005. "Aspectos claves de la colaboración”. En Alianzas sociales en América Latina: enseñanzas extraídas de colaboraciones entre el sector privado y organizaciones de la sociedad civil. 3-29. Banco Interamericano del Desarrollo.

Brammer, Stephen, Andrew Millington \& Sthephen Pavelin. 2006. "Is Philanthropy Strategic? An Analysis of the Management of Charitable Giving in Large UK Companies". Journal of Business Ethics 15 (3): 234-245.

Carroll, Archie. 1991. "The Pyramid of Corporate Social Responsibility: Toward the Moral Management of Organizational Stakeholders". Business Horizons 34 (4): 39-48.

Carroll, Archie \& Kareem Shabana. 2010. "The Business Case for Corporate Social Responsibility: A Review of Concepts, Research and Practice". International Journal of Management Reviews 12 (1): 85-105.

Chen, Jennifer, Dennis Patten \& Robin Roberts. 2008. "Corporate Charitable Contributions: A Corporate Social Performance or Legitimacy Strategy?". Journal of Business Ethics 82: 131-144.

Eccles, Robert, Ioannis Ioannou \& George Serafeim. 2011. "The Impact on Corporate Sustainability on Organizational Processes and Performance". Management Science 60 (11): 2835-2857.

File, Karen \& Russ Prince. 1998. "Cause Related Marketing and Corporate Philanthropy in the Privately Held Enterprise". Journal of Business Ethics 17 (14): 1529-1539.

Friedman, Milton. 1970. "The Social Responsibility of Business is to Increase its Profits". The New York Times Magazine, 13 de septiembre.

Godfrey, Paul. 2005. "The Relationship between Corporate Philanthropy and Shareholder Wealth: A Risk Management Perspective". Academy of Management Review 30 (4): 777-798. 
Irarrázaval, Ignacio, Paula Streeter, Gastón Fernández \& Carolina Hazbún. 2017. Fortaleciendo la sociedad civil: el rol de los incentivos tributarios en las donaciones. Santiago: Centro de Políticas Públicas UC y Fundación Chile Más Hoy.

Kurucz, Elizabeth, Barry Colbert \& David Wheeler. 2008. "The Business Case for Corporate Social Responsibility". En The Oxford Handbook of Corporate Social Responsibility, editado por Andrew Crane, Dirk Matten, Abagail McWilliams, Jeremy Moon \& Donald S. Siegel. Oxford: Oxford University Press.

Maas, Karen \& Kellie Liket. 2010. "Talk the Walk: Measuring the Impact of Strategic Philanthropy”. Journal of Business Ethics 100: 445-464.

Porter, Michael, 1990. "The Competitive Advantage of Nations". Harvard Business Review (marzo-abril): 73-91.

Porter, Michael \& Michael Kramer.1999. "Philanthropy’s New Agenda: Creating Value". Harvard Business Review (November-December): 121-130.

- 2002. "The Competitive Advantage of Corporate Philanthropy". Harvard Business Review 80 (12): Reprint R0212D 1-14.

- 2006. "Strategy and Society: The Link between Competitive Advantage and Corporate Social Responsibility". Harvard Business Review 84 (12): Reprint R0612D 1-13.

- 2011. "Creating Shared Value". Harvard Business Review (enero-febrero): 3-18.

Post, James \& Sandra Waddock. 1995. "Strategic Philanthropy and Partnerships for Economic Progress". En Philanthropy and Economic Development, editado por Richard F. America. Westport: Greenwood Press.

Saiia, David, Archie Carrol \& Ann Buchholtz. 2003. "Philanthropy as Strategy: When Corporate Charity 'Begins at Home"'. Business and Society 42: 169201.

Schwartz, Mark \& Archie Carroll. 2008. "Integrating and Unifying Competing and Complimentary Frameworks: The Search for a Common Core in the Business and Society Field". Business and Society 47: 148-186.

Smith, N. Graig. 2003. "Corporate Social Responsibility: Whether or How?" California Management Review 45: 52-76.

Thorne, Debbie \& Linda Ferrell. 2002. "The Role of Strategic Philanthropy in Marketing Strategy". European Journal of Marketing 36 (5): 689-705.

Van Marrewijk, Marcel. 2003. "Concepts and Definitions of CSR and Corporate Sustainability: Between Agency and Communion”. Journal of Business Ethics 44: 95-105.

Van Marrewijk, Marcel \& Marco Werre. 2003. "Multiple Levels of Corporate Sustainability". Journal of Business Ethics 44: 107-119.

Wang, Heli, Jaepil Choi \& Jiatao Li. 2008. “Too Little or Too Much? Untangling the Relationship between Corporate Philanthropy and Firm Financial Performance". Organization Science 19: 143-159. EP 